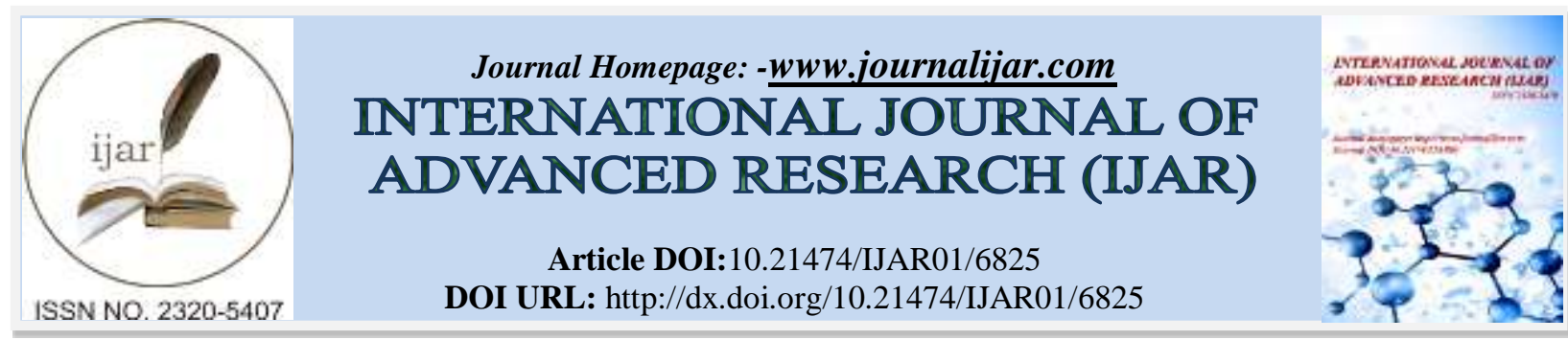

RESEARCH ARTICLE

\title{
REVIEW ON HEALTH RELATED QUALITY OF LIFE (HRQOL) OF PATIENTS AFTER STROKE.
}

\author{
Dr. Lukima Saikia.
}

PhD, M.Sc (N), PGDHM, Scottish Nurse Education Training (Napier University, UK), Lecturer, Regional College of Nursing, Guwahati, Assam.

\section{Manuscript Info}

Manuscript History

Received: 02 February 2018

Final Accepted: 04 March 2018

Published: April 2018

\section{Keywords:-}

Health related Quality of life ((HRQoL), Survivors, Impact, Stroke, Quality of Life (QOL), Apparently healthy adults (AHAs), National Institutes of Health stroke scale (NIHSS), Stroke-specific quality-of-life (SSQOL).

\begin{abstract}
Health-related quality of life (HRQoL) is a multi-dimensional concept that includes domains related to physical, mental, emotional, and social functioning. It goes beyond direct measures of population health, life expectancy, and causes of death. At this present scenario life expectancy continues to increase, the rates of chronic disease will also continue to grow. Patient-centric outcomes that include HRQoL will become even more important as individuals adjust to living with chronic conditions such as heart failure (HF) and stroke. Stroke is a chronic neurological disease with immense impact on the patients' lives. According to Centers for Disease control and prevention (CDC) 2018 February, Stroke is the fifth leading cause of death in the United States and is a major cause of serious disability for adults. About 795,000 people in the United States have a stroke each year. Stroke is one of the leading causes of death and disability in India. The estimated adjusted prevalence rate of stroke range, 84-262/100,000 in rural and 334-424/100,000 in urban areas. The incidence rate is 119$145 / 100,000$ based on the recent population based studies. The objective of this review was to explore the health related quality of life of patients after stroke and what are the impacts of stroke.
\end{abstract}

Copy Right, IJAR, 2018,. All rights reserved.

\section{Introduction:-}

Strokes are the second death of cause in the world. Projections show that one in every six people will have a stroke in life; 15 million people suffer a stroke per year and 6 million do not survive. Among those who do survive after stroke, most have residual impairments. Stroke remains a major contributor to mortality and morbidity both locally and globally. Stroke is a complex neurological illness which is commonly associated with multiple and long standing impairments. Although reduction of stroke mortality success in the care of individuals with a stroke, managing post-stroke consequences in those who survive remains a challenge. Survival from stroke frequently means living with some forms of disabilities. The number of people with stroke-related physical disabilities is escalating worldwide parallel to the growth of ageing population and increased lifestyle diseases such as hypertension and diabetes, which are known risk factors for stroke.

Impact of stroke on HRQoL:-

Jipan Xie, Zhi-Jie Zheng (Stroke. 2006 ;) reported Stroke is a major cause of long-term disability in the United States. This study examined the national impact of stroke on health-related quality of life (HRQoL) and disparities 
in HRQoL across different demographic groups. The study included 1040 non institutionalized stroke survivors. After adjustment for socio demographics, risk factors, and comorbidities, stroke survivors had statistically significantly lower mean scores for mental health $(4.1 \%)$, physical health $(7.9 \%)$, health utility $(6.9 \%)$, and selfrated health $(7.2 \%)$ (P0.01). In general, stroke did not affect differences in HRQoL among age or gender groups. However, racial and ethnic disparities in HRQoL were greater among stroke survivors than non-stroke individuals, particularly in health utility scores for black vs. white participants (0.06 in stroke survivors and 0.02 in the nonstroke population, P0.01) and Hispanic versus non-Hispanic participants $(0.11$ in stroke survivors and 0.01 in the non-stroke population).

Study concluded that Stroke significantly impairs HRQoL in the United States. The findings suggest that racial and ethnic disparities in HRQoL among stroke survivors are more pronounced than in the non-stroke population. The burden of nonfatal stroke, especially among racial and ethnic minorities, should be recognized more widely.

\section{Health-Related Quality of Life in Stroke Survivors:-}

According to the Jamaica health and lifestyle survey done in 2007, Stroke remains a major contributor to mortality and morbidity both locally and globally. Study was aimed to look at QOL among Jamaican stroke survivors across the vast spectrum of stroke severity ranging from mild to severe.

This was a hospital-based case-control study comparing the QOL of 50 stroke patients admitted to the University Hospital of the West Indies (UHWI) in Jamaica between 2012 and 2013 to that of 50 apparently healthy adults (AHAs). Stroke severity was calculated using the National Institutes of Health stroke scale (NIHSS) at admission. The health-related quality of life in stroke patients (HRQOLISP) tool was used to attain the QOL score in each group.

Study found that out of 108 patients that were coded as having an acute ischemic stroke in the year 2012, 38 (35.1\%) were deceased and $20(18.5 \%)$ were lost to follow-up at the time of the study. There was no statistical difference between the stroke group and the AHAs with regard to age, race and traditional risk factors for stroke. Within the stroke population, the mean age was $61 \pm 17.8$ years. The mean age among the AHAs was $60 \pm 13.1$ years. Of the stroke survivors, $25(50 \%)$ were male and $25(50 \%)$ were female. Forty-five (90\%) patients were hypertensive. Health-related quality of life (HRQOL) was significantly reduced across most domains when compared to AHAs (P $=0.0001)$. Greater stroke severity, presence of depression and previous stroke were all significantly associated with worse QOL.

In Jamaica, HRQOL among stroke survivors at the UHWI is consistently and significantly lower than that of healthy adults. Strategic interventions that target stroke severity, depression and non-adherence to secondary prevention regimens must be implemented in order to improve patient outcomes (Kerice Pinkney, Sandra Pinkney)

Mahran SA, Abdulrahman MA(2015),reported about the health-related quality of life in stroke survivors: clinical, functional, and psychosocial correlate.

The aims of this study were to examine the health-related quality of life (HRQOL) in stroke survivors attending an outpatient rehabilitation clinic and to examine the relation between some clinical variables and HRQOL.

Sixty-four stroke survivors were enrolled. Demographic data were obtained using a structured questionnaire. The functional level of the patients was assessed using the Barthel Index (BI). HRQOL was measured by the short-form 36 (SF-36) and the stroke-specific quality-of-life (SSQOL) scale.

Study found that patients' ages ranged from 42 to 95 years (mean 60.81 years), and men represented $81.2 \%$ of the participants. The 12 domains of SSQOL showed different degrees of deterioration in their means. Statistically significant differences between the means of the mobility level, and upper-limb and lower-limb voluntary control and SSQOL were found in favor of functional independency and full control of the limbs $(\mathrm{P}<0.05)$. Voluntary control of the limbs and BI showed a significant positive correlation with both SSQOL and the physical component of SF-36. Multiple regression analysis showed that the BI score, the nature of stroke, and the comorbidities are the most significant predictors of SSQOL, with P values of $0.004,0.013$, and 0.047 , respectively. 
It was concluded that HRQOL is impaired in stroke survivors, and that the functional independency level is its most significant predictor. They recommend considering the assessment of HRQOL in stroke survivors undergoing rehabilitation management as it is more relevant to the patients.

Health-related quality of life among chronic stroke survivors:-

Kong K H, Yang S Y revealed that study was conducted to assess health-related quality of life (HRQOL) among chronic stroke survivors and factors associated with it.

Cross-sectional survey study of patients who had survived one year or more after a stroke. Subjects consisted of stroke patients attending the outpatient clinic of a rehabilitation center. HRQOL was assessed using the Medical Outcomes 36-Item Short Form Health Survey (SF-36), functional status using the Modified Barthel Index (MBI), and mood using the Beck's Depression Inventory (BDI).

A total of 100 patients (63 men and 37 women, mean age $60.7+/-10.8$ years) were interviewed at an average of $22.2+/-14.5$ months post-stroke. The mean MBI was $88.9+/-17.9$, and the prevalence of depression $24 \%$. 50 percent of patients needed varying degrees of care for their activities of daily living. The SF-36 scores of the patients were comparable to that of the general population except for the domain of physical function, which was much lower. Age, sex, post-stroke duration, MBI score and side of hemiplegia were not associated with HRQOL. Depressed patients however had significantly lower SF-36 scores across all domains except for that of physical function and bodily pain (p-value is less than 0.001 ).

Study concluded that a significant proportion of chronic stroke survivors attending the rehabilitation clinic continue to face limitations in their physical activities. In addition, almost 30 percent of these survivors have depression that affects their HRQOL adversely.

\section{Measuring health-related quality of life after stroke: A Brief Tool:-}

Russell, M., Dempster, M., \& Donnelly, M. (2011) stated that Routine assessment of health-related quality of life (HRQoL) can be time consuming and burdensome for a person with stroke. Therefore the aim of this study was to develop and test a brief instrument for assessing HRQoL among people with stroke. The Quality of Life after Stroke Scale (QLASS) was constructed from items within the Quality of Life Index-Stroke Version and the Chronic Respiratory Disease Questionnaire. It was administered to 92 people with stroke at 3 points in time: immediately after discharge from hospital, 6 months and 12 months later. Results suggest that the QLASS has 19 items which represent 3 factors: emotional functioning, mastery and fatigue which correlate with valid measures of health status and activities of daily living. The QLASS is proposed as a brief, valid HRQoL tool for use among people with stroke.

In summary, this research suggests an alternative, brief instrument for assessing HRQoL among people with stroke, which will assist clinicians wishing to complete routine assessments of HRQoL and provide a useful tool to be used in research studies within this population

Health-related quality of life after stroke:-

The study was aimed to assess health-related quality of life after stroke and associate this event with the characteristics of people who suffered from stroke.

A cross-sectional study was conducted between February and August, 2014, at the rehabilitation center Centro Integrado de Reabilitação de Teresina, in the state of Piauí, northeast Brazil. This center is a non-profit institution dedicated to the rehabilitation of disabled people for stroke survivors with 104 patients. The Mini-mental state examination (MMSE); an instrument for collection of socio demographic, economic, clinical, and family related information; and the Stroke Specific Quality of Life Scale were applied.

Of the 104 people investigated, 77 did not have cognitive deficit and answered the instruments. Their mean at the MMSE was $24.9( \pm 4.3) .51 .9 \%$ were men and their mean age was $57.3( \pm 17.2)$ years. Most of them were married (48.1\%) and had eight or more years of schooling (50.7\%). Their health-related quality of life was affected (146.8 \pm 36.3$)$, mainly in the social and family relations domains. 
Study concluded that Health-related quality of life specifically among people who suffered a stroke decreases after its occurrence and is associated with some characteristics. Lower schooling, additionally to the occurrence of dyslipidemia, left-sided hemiplegia, and speech impairments contributed for its damaging. Higher scores on the MMSE, however, were related to better scores in terms of quality of life.

\section{Conclusion:-}

Stroke causes sufficient decrease in quality of life even among those who have no post stroke disability. According to Barclay \& Tate's prospective study carried out in 2014, stroke patients present a change in the responses observed over time, which they interpret as changes in life priorities according to each individual's post-stroke adaptation process. This alters the results in the quality of life self-assessment carried out by patients over time. Stroke has a major impact on survivors including Health related Quality of life (HRQoL). HRQoL measurements are potentially more relevant to patients than measurements of impairments or disability and are an important index of outcome after stroke that can facilitate a broader description of disease and outcome.

\section{References:-}

1. Ogunniyi A, Truelsen T, Bonita R, et al. Standard method for developing stroke registers in low-income and middle income countries: Experiences from a feasibility study of a stepwise approach to stroke surveillance (STEPS Stroke) Lancet Neurol. 2007;6:134-139. [PubMed]

2. Mayo NE, Wood-dauphine S, Ahmed S, et al. Disablement following stroke. Disabil Rehabil. 1999;21:258268. [PubMed]

3. Abubakar SA, Yunusa GH, Isezuo SA. Predictors of 30 days outcome of patients with acute stroke in Sokoto. Sahel Medical Journal. 2010;13(2):68-73.

4. Wahab K, Okubadejo N, Ojini F, Danesi M. Effect of admission hyperglycaemia on short-term outcome in adults Nigerians with first-ever acute ischaemic stroke. AJNS. 2007;26(2)

5. Owolabi MO. Determinants of Health-Related quality of life in Nigerian stroke survivors. Trans. R. Soc. Trop. Med. Hyg. 2008;102:1219-1250. [PubMed]

6. Lai MS, Studenski S, Duncan PW, Perera S. Persisting consequences of stroke measured by the stroke impact scale. Stroke. 2002;33:1840-1844. [PubMed]

7. Rochette A, Desrosiers J, Norrau L. Associated between personal and environmental factors and occurrence of handicap situation following a stroke. Disabil Rehabil. 2001;23:559-569. [PubMed]

8. Sturm JW, Donnan GA, Dewey HM, Macdonell RA, et al. Determinants of handicap after stroke. North East Melboure Stroke Incidence Study. Stroke. 2004;35:715-720. [PubMed]

9. Carod-Artel J, Egido JA, Gonzalez JL, de Seijas EV. Quality of life among stroke survivors evaluated 1 year stroke. Experience of a stroke unit. Stroke. 2000;31:2995-3000. [PubMed]

10. Meckenzie AE, Chang AM. Predictors of Quality of life following stroke. Disabil Rehabil. 2002;24:259-265. [PubMed]

11. Suenkeler IH, Nowak M, Misselwiz B, Kugler C, et al. Time course of Heath related Quality of Life as determined $36 \& 12$ months after stroke [PubMed]

12. Lavados PM, Hennis AJ, Fernandes JG, Medina MT, Legetic B, Hoppe A, et al. Stroke epidemiology, prevention, and management strategies at a regional level: Latin America and the Caribbean. Lancet Neurol $2007 ; 6: 362-372$.

13. Mar J, Sainz-Ezkerra M, Moler-Cuiral JA. Calculation of prevalence estimates through differential equations: application to stroke-related disability. Neuroepidemiology. 2008; $31: 57-66$.

14. Wilkinson PR, Wolfe CDA, Warburton FG, Rudd AG, Howard RS, Ross-Russell RW, Beech RR, et al. A longterm follow up of stroke patients. Stroke 1997; 28 :507-512.

15. Bush TL, Miller SR, Golden AL, Hale WE. Self-report and medical record report agreement of selected medical conditions in the elderly. Am J Public Health 1989; 79 :1554-1556.

16. Williams LS, Weinberger M, Harris LE, Biller J. Measuring quality of life in a way that is meaningful to stroke patients. Neurology 1999; $53: 1839-1843$.

17. Lima RCM, Teixeira-Salmela LF, Magalhaes LC, Gomes-Neto M. Psychometric properties of the Brazilian version of the Stroke Specific Quality of Life Scale: application of the Rasch model. Rev Bras Fisioter 2008; 12 :149-156.

18. Muus I, Williams LS, Ringsberg KC. Validation of the Stroke Specific Quality of Life Scale (SS-QOL): test of reliability and validity of the Danish version (SS-QOL-DK). Clin Rehabil 2007; 21:620-627. 
19. Ewert T, Stucki G. Validity of the SS-QOL in Germany and in survivors of hemorrhagic or ischemic stroke. Neurorehabil Neural Repair 2007; $21: 161-168$.

20. Fróes KS, Valdés MT, Lopes Dde P, Silva CE. Factors associated with health-related quality of life for adults with stroke sequelae. Arq Neuropsiquiatr 2011; 69 :371-376.

21. Noble AJ, Schenk T. Which variables help explain the poor health-related quality of life after subarachnoid hemorrhage? A meta-analysis. Neurosurgery 2010; $66: 772-783$.

22. Carod-Artal FJ, Egido JA. Quality of life after stroke: the importance of a good recovery. Cerebrovasc Dis 2009; 27(Suppl 1) :204-214.

23. Graham A. Measurement in stroke: activity and quality of life. In: Barnes MP, Dobkin BH, Bogusslavsky J, editors. Recovery after stroke. New York: Cambridge University Press; 2005. 135-160.

24. Chou CY. Determinants of the health-related quality of life for stroke survivors. J Stroke Cerebrovasc Dis 2015; $24: 655-662$. 